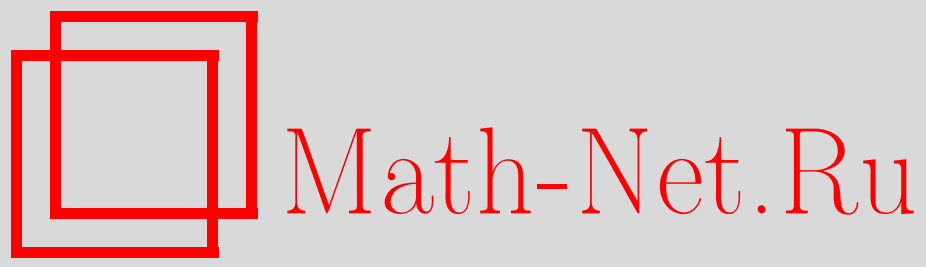

F. Götze, A. N. Tikhomirov, Rate of convergence to the semi-circular law for the Gaussian unitary ensemble, Теория вероятн. и ее примен., 2002, том 47, выпуск 2, 381-387

DOI: https://doi.org/10.4213/tvp3670

Использование Общероссийского математического портала MathNet.Ru подразумевает, что вы прочитали и согласны с пользовательским соглашением

http: //www . mathnet.ru/rus/agreement

Параметры загрузки:

IP : 35.173 .219 .12

26 апреля 2023 г., 16:00:12

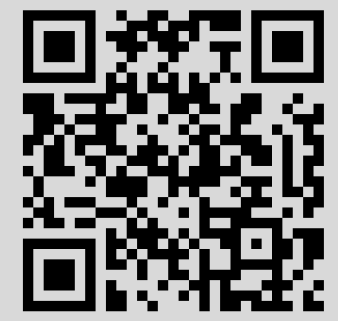


8. Чибисов Д.М. Асимптотическое разложение для распределения статистики, допускающей стохастическое разложение. I. - Теория вероятн. и ее примен., 1980, т. 25 , в. 4 , с. $745-756$.

9. Chung K.L. The approximate distribution of Student's statistics. - Ann. Math. Statist., 1946, v. 17, p. 447-465.

10. Esseen C.-G. Fourier analysis of distribution functions. A mathematical study of the Laplace-Gaussian law. - Acta Math., 1945, v. 77, p. 1-125.

11. Friedrich K.O. A Berry-Esseen bound for functions of independent random variables. - Ann. Statist., 1989, v. 17, № 1, p. 170-183.

12. Hall $P$. Edgeworth expansion for Student's $t$ statistic under minimal moment conditions. - Ann. Statist., 1987, v. 15, p. 920-931.

13. Hall $P$. On the effect of random norming on the rate of convergence in the central limit theorem. - Ann. Probab., 1988, v. 16, № 3, p. 1265-1280.

14. Helmers $R$. On the Edgeworth expansion and the bootstrap approximation for a Studentized $U$-statistic. - Ann. Statist., 1991, v. 19, № 1, p. 470-484.

15. Helmers $R$. The Berry-Esseen bound for Studentized $U$-statistics. - Canad. J. Statist., 1985 , v. 13 , p. $79-82$.

16. Helmers R., van Zwet W. The Berry-Esseen bound for $U$-statistics. - Statistical Decision Theory and Related Topics III: Proceedings of the Third Purdue Symposium, 1981, V. 1. Ed. by S. S. Gupta and J. O. Berger. New York: Academic Press, 1982, p. $497-512$.

17. Prašková $Z$. Sampling from a finite set of random variables: the Berry-Esseen bound for the Studentized mean. - Proceedings of the Fourth Prague Symposium on Asymptotic Statistics. Prague: Charles Univ., 1989, p. 67-82.

18. Putter H., van Zwet W.R. Empirical Edgeworth expansions for symmetric statistics. - Ann. Statist., 1998, v. 26, № 4, p. 1540-1569.

19. Singh $K$. On the asymptotic accuracy of Efron's bootstrap. - Ann. Statist., 1981, v. 9 , p. $1187-1195$.

20. Slavova $V$. V. On the Berry-Esseen bound for Student's statistic. - Lecture Notes in Math., 1985, v. 1155, p. 335-390.

Поступила в редакцию 13.V.1999

(C) 2002 г. GÖTZE F.*, TIKHOMIROV A. N.**

\title{
RATE OF CONVERGENCE TO THE SEMI-CIRCULAR LAW FOR THE GAUSSIAN UNITARY ENSEMBLE ${ }^{1)}$
}

\begin{abstract}
Показано, что расстояние Колмогорова между усредненным значением спектральной функции распределения матрицы Вигнера размера $n \times n$ с гауссовскими элементами и функцией распределения полукругового закона имеет порядок $O\left(n^{-2 / 3}\right)$.
\end{abstract}

${ }^{*}$ Fakultät für Mathematik, Universität Bielefeld, 33501 Bielefeld 1, Germany; e-mail: goetze@mathematik.uni-bielefeld.de

** Сыктывкарский государственный университет, Октябрьский проспект., 55, 167001 Сыктывкар, Россия; e-mail: tikhomir@ssu.komi.com

1) Research of both authors supported by the DFG-Forschergruppe FOR 399/1-1 «Spektrale Analysis, Asymptotische Verteilungen und Stochastische Dynamiken». The second author partially supported by Russian Foundation for Basic Research (Grants № 99-01-00247, 99-01-00112, 00-15-96019) and by INTAS № 99-01317, DFG-RFBR № 9901-04027. 
Ключевые слова и фразы: независимые случайные величины, спектральное распределение, случайная матрица.

1. Introduction and results. Let $W_{n}$ be a Hermitean matrix of order $n$ whose elements $W_{l j}=X_{l j}+i Y_{l j}(l, j=1, \ldots, n)$ are independent (apart from the conditions of being Hermitean). Assume that the real and imaginary parts of matrix elements are independent as well. Furthermore let $\mathbf{E} X_{l j}=0$ and $\mathbf{E} Y_{k j}=0,1 \leqslant l \leqslant j$ and $1 \leqslant k<j$. Finally we may assume that $\mathbf{E} X_{l l}^{2}=2 \sigma^{2}, l=1,2, \ldots$, and $\mathbf{E} X_{k j}^{2}=\mathbf{E} Y_{k j}^{2}=\sigma^{2}, 1 \leqslant k<j$. Let $Y_{l l}=0$ for $l=1,2, \ldots$.

Let $\lambda_{1}, \lambda_{2}, \ldots, \lambda_{n}$ denote the eigenvalues of the random matrix $n^{-1 / 2} W$ and define the spectral distribution function of this matrix by

$$
F_{n}(x)=\frac{1}{n} \sum_{j=1}^{n} \mathbf{I}_{(-\infty, x]}\left(\lambda_{j}\right),
$$

where $\mathbf{I}_{(a, b]}(u)$ denotes the indicator function of the interval $(a, b]$. For simplicity we shall assume that $\sigma^{2}=\frac{1}{4}$. Denote by $G(x)$ the distribution function of the semi-circular law with density $g(x)=G^{\prime}(x)=\pi^{-1} \sqrt{2-x^{2}} \mathbf{I}_{[-\sqrt{2}, \sqrt{2}]}(x)$. Wigner [13], [14] showed that

$$
\Delta_{n}:=\sup _{x}\left|\mathbf{E} F_{n}(x)-G(x)\right| \rightarrow 0 \text { as } n \rightarrow \infty,
$$

under assumption that the random variables $X_{j k}$ and $Y_{j k}$ have finite moments. Pastur [12] has proven that $F_{n}(x)$ tends to $G(x)$ in probability when Lindeberg's condition is fulfilled for every row vector of matrix $W_{n}$. Girko [5] proved that if $\lim _{n \rightarrow \infty} n^{-2} \sum_{l, j=1}^{n} \mathbf{E}\left|W_{l j}\right|^{2} \mathbf{I}_{\left\{\left|W_{l j}\right|>\tau \sqrt{n}\right\}}=0$, for some $\tau>0$, then $F_{n}(x) \rightarrow G(x)$ a.s. as $n \rightarrow \infty$. The actual rate of convergence in limit theorems of type (1.1) is unknown.

Girko [7] obtained the bound $\Delta_{n}=O\left(n^{-1 / 14}\right)$. Bai [1] has proven that $\Delta_{n}=O\left(n^{-1 / 4}\right)$ assuming that $M:=\sup _{l, j} \mathbf{E}\left|W_{l, j}\right|^{4}<\infty$ and in [3] improved this result to $\Delta_{n}=O\left(n^{-1 / 3}\right)$. Götze and Tikhomirov [8] proved that $\Delta_{n} \leqslant C M^{1 / 2} n^{-1 / 2}$. Bai [2] announced the bound $\Delta_{n}=O\left(n^{-1 / 2}\right)$ and Girko [6] stated this bound, but it seems that in his proof some critical steps concerning the Gaussian unitary ensemble (GUE) are not correct. A closer analysis of that GUE case motivated our paper. We investigate the rate of convergence of the expected normalized spectral distribution function to the semi-circular law for Gaussian random variables $X_{l j}$ and $Y_{k j}$. We shall consider nonuniform bounds as well as uniform ones. Throughout this note we shall assume that $X_{l j}, 1 \leqslant l \leqslant j$, and $Y_{k j}, 1 \leqslant k<j$, are independent Gaussian random variables with zero mean and $\sigma^{2}=\frac{1}{4}$. The main results are the following.

Theorem 1.1. There exist positive absolute constants $C_{1}, C$ such that for any $x \in$ $\left[-\sqrt{2}+C_{1} n^{-1 / 3}, \sqrt{2}-C_{1} n^{-1 / 3}\right]$ the following inequality holds for the GUE ensemble:

$$
\left|\mathbf{E} F_{n}(x)-G(x)\right| \leqslant \frac{C}{n\left(2-x^{2}\right)} .
$$

Theorem 1.2. There exists a positive absolute constant $C$ such that for the GUE ensemble

$$
\Delta_{n} \leqslant C n^{-2 / 3} \text {. }
$$

2. Asymptotics of Hermitean orthogonal polynomials. Let $\varphi_{n}(x)$ denote the $n$-th Hermite function, i.e.,

$$
\varphi_{n}(x)=\frac{1}{\sqrt{n ! 2^{n} \sqrt{\pi}}} e^{-x^{2} / 2} H_{n}(x),
$$

where $H_{n}(x)$ the $n$-th Hermite polynomial, $H_{n}(x)=e^{x^{2}}\left(-\frac{d}{d x}\right)^{n} e^{-x^{2}}$. In what follows we shall denote by $\theta$ some quantity, possible depending on $n$ and $x$, such that $|\theta| \leqslant 1$. In this section we recall the asymptotics of $\varphi_{n}(x)$. We have the following asymptotic property of Hermitean functions. Let $N=2 n+1$. Then

$$
\varphi_{n}(x)=\left(\frac{2}{\pi}\right)^{1 / 2}\left(N-x^{2}\right)^{-1 / 4} \cos \left(\frac{N(2 \beta-\sin 2 \beta)-\pi}{4}\right)+O\left(N^{1 / 2}\left(N-x^{2}\right)^{-7 / 4}\right) \text {, }
$$


where $0 \leqslant x \leqslant N^{1 / 2}$ and $\beta:=\beta_{n}(x)=\arccos \left(x N^{-1 / 2}\right)$. For the proof of this equality see $[4]$ and $[11]$.

We rewrite this result as follows.

Corollary 2.1. There exists some absolute positive constant $C$ such that, for any $0 \leqslant x \leqslant \sqrt{2}-C n^{-1 / 3}$, the following equality holds:

$$
\begin{aligned}
n^{1 / 4} \varphi_{n}(x \sqrt{n})= & \left(\frac{2}{\pi}\right)^{1 / 2}\left(2-x^{2}\right)^{-1 / 4} \cos \left\{\frac{n}{2}\left(2 \beta_{0}-\sin \left(2 \beta_{0}\right)\right)+\frac{\beta_{0}}{2}-\frac{\pi}{4}\right\} \\
& +\theta \frac{C}{n\left(2-x^{2}\right)^{7 / 4}},
\end{aligned}
$$

where $\beta_{0}:=\beta_{0}(x)=\arccos (x / \sqrt{2})$.

$\mathrm{P} \mathrm{r}$ o o f. At first we note that

$$
\left(N-x^{2} n\right)^{-1 / 4}=n^{-1 / 4}\left(2-x^{2}\right)^{-1 / 4}\left(1+O\left(\frac{1}{n\left(2-x^{2}\right)}\right)\right) .
$$

Furthermore, using Taylor's expansion for the function $\arccos y$ we obtain

$$
\beta(x \sqrt{n})=\arccos \left(x \sqrt{\frac{n}{2 n+1}}\right)=\arccos \left(\frac{x}{\sqrt{2}}\right)+\frac{1}{4 n} \frac{\cos \beta_{0}}{\sin \beta_{0}}+O\left(\frac{1}{n^{2}\left(2-x^{2}\right)^{3 / 2}}\right) .
$$

Analogously we obtain

$$
\sin (2 \beta(x \sqrt{n}))=\sin \left(2 \beta_{0}\right)+\frac{1}{2 n} \frac{\cos \beta_{0}}{\sin \beta_{0}} \cos \left(2 \beta_{0}\right)+O\left(\frac{1}{n^{2}\left(2-x^{2}\right)^{3 / 2}}\right) .
$$

The equalities (2.4) and (2.5) together imply

$$
\frac{2 n+1}{4}(2 \beta-\sin (2 \beta))=\frac{n}{2}\left(2 \beta_{0}-\sin \left(2 \beta_{0}\right)\right)+\frac{\beta_{0}}{2}+O\left(\frac{1}{n\left(2-x^{2}\right)^{3 / 2}}\right) .
$$

Substituting (2.3) and (2.6) in (2.1) we obtain the result of Corollary 2.1.

3. Non-uniform bound. The proof of Theorem 1.1. Let $\sigma_{n}(x)$ denote the density of the expected normalized spectral distribution function of a Gaussian Hermitean matrix $W$. It is well known that

$$
\begin{aligned}
\sigma_{n}(x) & =\frac{d}{d x} \mathbf{E} F_{n}(x)=\frac{1}{\sqrt{n}} \sum_{k=0}^{n-1} \varphi_{k}^{2}(x \sqrt{n}) \\
& =\sqrt{n} \varphi_{n}^{2}(x \sqrt{n})-\sqrt{n+1} \varphi_{n-1}(x \sqrt{n}) \varphi_{n+1}(x \sqrt{n})
\end{aligned}
$$

(see, for example, [10, Appendix A.8]). Similarly to Girko's [6] approach we write

$$
(n+1)^{1 / 4} \varphi_{n+1}(x \sqrt{n})=(n+1)^{1 / 4} \varphi_{n+1}\left(x \sqrt{n+1} \sqrt{\frac{n}{n+1}}\right) .
$$

According to Corollary 2.1 we get

$$
\begin{aligned}
(n+1)^{1 / 4} \varphi_{n+1}(x \sqrt{n})= & \left(\frac{2}{\pi}\right)^{1 / 2}\left(2-x^{2}\right)^{-1 / 4} \cos \left\{\frac{n}{2}\left(2 \beta_{0}-\sin \left(2 \beta_{0}\right)\right)+\frac{3 \beta_{0}}{2}-\frac{\pi}{4}\right\} \\
& +\theta \frac{C}{n\left(2-x^{2}\right)^{7 / 4}} .
\end{aligned}
$$

Analogously we obtain

$$
\begin{aligned}
(n+1)^{1 / 4} \varphi_{n-1}(x \sqrt{n})= & \left(\frac{2}{\pi}\right)^{1 / 2}\left(2-x^{2}\right)^{-1 / 4} \cos \left\{\frac{n}{2}\left(2 \beta_{0}-\sin \left(2 \beta_{0}\right)\right)-\frac{\beta_{0}}{2}-\frac{\pi}{4}\right\} \\
& +\theta \frac{C}{n\left(2-x^{2}\right)^{7 / 4}} .
\end{aligned}
$$


The equalities (3.1)-(3.3) and (2.2) together imply that

$$
\begin{aligned}
\sigma_{n}(x)=\frac{2}{\pi \sqrt{2-x^{2}}}[ & \cos ^{2}\left\{\frac{n}{2}\left(2 \beta_{0}-\sin \left(2 \beta_{0}\right)\right)-\frac{\pi}{4}+\frac{\beta_{0}}{2}\right\} \\
& -\cos \left\{\frac{n}{2}\left(2 \beta_{0}-\sin \left(2 \beta_{0}\right)\right)-\frac{\pi}{4}-\frac{\beta_{0}}{2}\right\} \\
& \left.\times \cos \left\{\frac{n}{2}\left(2 \beta_{0}-\sin \left(2 \beta_{0}\right)\right)-\frac{\pi}{4}+\frac{3 \beta_{0}}{2}\right\}\right]+\theta \frac{C}{n\left(2-x^{2}\right)^{2}} \\
=\frac{2}{\pi \sqrt{2-x^{2}}} \sin ^{2} \beta_{0} & +\theta \frac{C}{n\left(2-x^{2}\right)^{2}}=\frac{\sqrt{2-x^{2}}}{\pi}+\theta \frac{C}{n\left(2-x^{2}\right)^{2}} .
\end{aligned}
$$

This implies that, uniformly for $x \in\left[-\sqrt{2}+C_{1} n^{-1 / 3}, \sqrt{2}-C_{1} n^{-1 / 3}\right]$,

$$
\left|\sigma_{n}(x)-g(x)\right| \leqslant \frac{C}{n\left(2-x^{2}\right)^{2}} .
$$

Here $g(x)$ denote the density of the semi-circular law, $g(x)=\pi^{-1} \sqrt{2-x^{2}} \mathbf{I}_{[-\sqrt{2}, \sqrt{2}]}(x)$. Since $\mathbf{E} F_{n}(0)=G(0)$ we get

$$
\left|\mathbf{E} F_{n}(x)-G(x)\right| \leqslant \frac{C}{n} \int_{0}^{|x|} \frac{d u}{\left(2-u^{2}\right)^{2}} \leqslant \frac{C}{n\left(2-x^{2}\right)} .
$$

This implies that, for $x \in\left[-\sqrt{2}+C_{1} n^{-1 / 3}, \sqrt{2}-C_{1} n^{-1 / 3}\right]$,

$$
\left|\mathbf{E} F_{n}(x)-G(x)\right| \leqslant \frac{C}{n\left(2-x^{2}\right)} \leqslant \frac{C}{n^{2 / 3}}
$$

and completes the proof.

4. The uniform bound and proof of Theorem 1.2. To obtain the uniform bound we consider now the intervals

$$
\begin{aligned}
\mathbf{J}_{\eta}^{(l)} & =[-\sqrt{2},-\sqrt{2}+\eta], & & \mathbf{J}_{\eta}^{(r)}=[\sqrt{2}-\eta, \sqrt{2}], \\
\mathbf{J}_{\varepsilon}^{(l)} & =[-\sqrt{2}+\varepsilon,-\sqrt{2}+\eta], & \mathbf{J}_{\varepsilon}^{(r)} & =[\sqrt{2}-\eta, \sqrt{2}-\varepsilon], \\
\mathbf{J}_{\varepsilon}^{\prime(l)} & =[-\sqrt{2},-\sqrt{2}+\eta+\varepsilon], & \mathbf{J}_{\varepsilon}^{\prime(r)} & =[\sqrt{2}-\eta-\varepsilon, \sqrt{2}] .
\end{aligned}
$$

Consider the distance $\Delta_{\eta}:=\sup _{x \in \mathbf{J}_{\eta}^{(r)} \cup \mathbf{J}_{\eta}^{(l)}}|F(x)-G(x)|$. In what follows we shall assume that

$$
\Delta:=\sup _{x}|F(x)-G(x)|=\Delta_{\eta}
$$

Lemma 4.1. Let $F$ be a distribution function and let $G$ denote the semi-circular distribution function. Denote their Stieltjes transforms by $f(z)$ and $g(z)$ respectively, where $z=u+i v$. Assume that $\int|F(x)-G(x)| d x<\infty$ and the condition (4.1) holds. Let $v>0$ and let $a$ and $\eta$ be positive numbers such that

$$
\begin{gathered}
\gamma=\frac{1}{\pi} \int_{|u| \leqslant a} \frac{1}{u^{2}+1} d u>\frac{3}{4}, \\
\eta \geqslant 2 \varepsilon \geqslant 2 v a .
\end{gathered}
$$

Then there exist some positive constants $C_{1}(\gamma), C_{2}(\gamma), C_{3}(\gamma)$, depending on $\gamma$ such that

$$
\Delta_{\eta} \leqslant C_{1}(\gamma) \sup _{x \in \mathbf{J}_{\varepsilon}^{\prime}(r) \cup \mathbf{J}_{\varepsilon}^{\prime(l)}}\left|\operatorname{Im}\left(\int_{-\infty}^{x}(f(z)-g(z)) d u\right)\right|+C_{2}(\gamma) v \sqrt{\eta}+C_{3}(\gamma) \varepsilon^{3 / 2} .
$$


P r o o f. Introduce the following notation $\Delta_{\varepsilon}:=\sup _{x \in \mathbf{J}_{\varepsilon}^{(r)} \cup \mathbf{J}_{\varepsilon}^{(l)}}|F(x)-G(x)|$. Note that $\Delta_{\eta} \leqslant \Delta_{\varepsilon}+C \varepsilon^{2 / 3}$. We have the following relations:

$$
\begin{aligned}
\frac{1}{\pi} & \operatorname{Im}\left(\int_{-\infty}^{x}(f(z)-g(z)) d u\right)=\frac{1}{\pi} \int_{-\infty}^{x}\left[\int_{-\infty}^{\infty} \frac{v d(F(y)-G(y))}{(y-u)^{2}+v^{2}}\right] d u \\
& =\frac{1}{\pi} \int_{-\infty}^{x}\left[\int_{-\infty}^{\infty} \frac{2 v(y-u)(F(y)-G(y)) d y}{\left((y-u)^{2}+v^{2}\right)^{2}}\right] d u \\
& =\frac{1}{\pi} \int_{-\infty}^{\infty}(F(y)-G(y))\left[\int_{-\infty}^{x} \frac{2 v(y-u) d u}{\left((y-u)^{2}+v^{2}\right)^{2}}\right] d y \\
& =\frac{1}{\pi} \int_{-\infty}^{\infty} \frac{(F(x-v y)-G(x-v y)) d y}{y^{2}+1} .
\end{aligned}
$$

Since $F$ is nonincreasing we obtain

$$
\begin{aligned}
& \frac{1}{\pi} \int_{|y|<a} \frac{(F(x-v y)-G(x-v y)) d y}{y^{2}+1} \geqslant \gamma(F(x-v a)-G(x-v a)) \\
& \quad-\frac{1}{\pi} \int_{|y|<a}|G(x-v y)-G(x-v a)| d y \\
& \geqslant \gamma(F(x-v a)-G(x-v a))-\frac{1}{v \pi} \int_{|y|<v a}|G(x-y)-G(x-v a)| d y .
\end{aligned}
$$

Replacing $x-v a$ by $x$ we get

$$
\begin{aligned}
& \frac{1}{\pi} \int_{|y|<a} \frac{(F(x+v a-v y)-G(x+v a-v y)) d y}{y^{2}+1} \\
& \quad \geqslant \gamma(F(x)-G(x))-\frac{1}{\pi} \int_{|y|<a}|G(x+v a-v y)-G(x+v a-v a)| d y \\
& \quad \geqslant \gamma(F(x)-G(x))-\frac{1}{v \pi} \int_{|y|<v a}|G(x+v a-y)-G(x+v a-v a)| d y .
\end{aligned}
$$

If (4.2) and (4.3) hold and $x \in \mathbf{J}_{\varepsilon}^{(r)} \cup \mathbf{J}_{\varepsilon}^{(l)}$ then $x \pm v a \in \mathbf{J}_{\varepsilon}^{\prime(r)} \cup \mathbf{J}_{\varepsilon}^{\prime(l)}$.

Let $x_{n} \in \mathbf{J}_{\varepsilon}^{(r)} \cup \mathbf{J}_{\varepsilon}^{(l)}$ be such that $F\left(x_{n}\right)-G\left(x_{n}\right) \rightarrow \Delta_{\eta}$. Then we have

$$
\begin{aligned}
\sup _{x \in \mathbf{J}_{\varepsilon}^{\prime}(r) \cup \mathbf{J}_{\varepsilon}^{\prime}(l)}\left|\frac{1}{\pi} \operatorname{Im}\left(\int_{-\infty}^{x}(f(z)-g(z)) d u\right)\right| \geqslant \gamma \lim _{n \rightarrow \infty}\left(F\left(x_{n}\right)-G\left(x_{n}\right)\right) \\
\quad-\frac{1}{v \pi} \sup _{x \in \mathbf{J}_{\varepsilon}^{\prime(r)} \cup \mathbf{J}_{\varepsilon}^{\prime(l)}} \int_{|y|<2 v a}|G(x+y)-G(x)| d y-(1-\gamma) \Delta_{\eta} \\
\geqslant \gamma \Delta_{\eta}-C a v \sqrt{\eta}-(1-\gamma) \Delta_{\eta}-C \varepsilon^{2 / 3} \geqslant(2 \gamma-1) \Delta_{\eta}-C a v \sqrt{\eta}-C \varepsilon^{2 / 3} .
\end{aligned}
$$

Similar arguments may be used for the sequence $x_{n} \in \mathbf{J}_{\varepsilon}^{(r)} \cup \mathbf{J}_{\varepsilon}^{(l)}$ such that $\left(F\left(x_{n}\right)\right.$ $\left.G\left(x_{n}\right)\right) \rightarrow-\Delta_{\eta}$. This completes the proof.

Lemma 4.2. For any $V>v_{0}>0$ the following inequality holds:

$$
\begin{aligned}
& \sup _{x \in \mathbf{J}_{\varepsilon}^{\prime(r)} \cup \mathbf{J}_{\varepsilon}^{\prime}(l)}\left|\int_{-\infty}^{x} \operatorname{Im}(f(z)-g(z)) d u\right| \leqslant \int_{-\infty}^{\infty}|f(u+i V)-g(u+i V)| d u \\
& +\sup _{x \in \mathbf{J}_{\varepsilon}^{\prime(r)} \cup \mathbf{J}_{\varepsilon}^{\prime(l)}} \mid \operatorname{Im}\left\{\int_{v_{0}}^{V}(f(x+i v)-g(x+i v)) d v\right\} .
\end{aligned}
$$

For the proof of this lemma see [8, Proof of Lemma 2.2].

Lemmas 4.1 and 4.2 together imply 
Corollary 4.1. There exist some absolute positive constants $C_{1}, C_{2}$ and $C_{3}$ such that for any $V>v_{0}>0$ and $\varepsilon>0$ such that $\eta \geqslant 2 \varepsilon \geqslant 2$ va the following inequality holds:

$$
\begin{aligned}
\Delta_{\eta} \leqslant & C_{1}\left(\int_{-\infty}^{\infty}|f(u+i V)-g(u+i V)| d u\right. \\
& \left.+\sup _{x \in \mathbf{J}_{\varepsilon}^{\prime(r)} \cup \mathbf{J}_{\varepsilon}^{\prime(l)}}\left|\operatorname{Im}\left\{\int_{v_{0}}^{V}(f(x+i u)-g(x+i u)) d u\right\}\right|\right) \\
& +C_{2} v_{0} \sqrt{\eta}+C_{3} \varepsilon^{2 / 3} .
\end{aligned}
$$

Let $W(k)$ be the matrix obtained from $W$ by deleting the $k$-th row and $k$-th column, and let $\alpha^{\prime}(k)=\left(X_{1 k}+i Y_{1 k}, \ldots, X_{(k-1) k}+i Y_{(k-1) k}, X_{(k+1) k}+i Y_{(k+1) k}, \ldots, X_{n k}+i Y_{n k}\right)$. Set

$$
\varepsilon_{k}=\frac{1}{\sqrt{n}} X_{k k}-\frac{1}{n} \overline{\alpha^{\prime}(k)}\left(W(k)-z \mathbf{I}_{n-1}\right)^{-1} \alpha_{k}+\frac{s_{n}(z)}{2},
$$

where $\mathbf{I}_{n-1}$ denotes the $(n-1) \times(n-1)$ identity matrix and $\bar{a}$ denotes the complex conjugate to a complex number $a$. Introduce

$$
\delta_{n}(z)=-\frac{1}{n} \sum_{k=1}^{n} \mathbf{E} \varepsilon_{k} \frac{1}{\left(z+s_{n}(z) / 2\right)\left(z+s_{n}(z) / 2-\varepsilon_{k}\right)} .
$$

Let

$$
s(z)=-\left(z-\sqrt{z^{2}-2}\right), \quad s_{n}(z)=\int_{-\infty}^{\infty} \frac{1}{x-z} d \mathbf{E} F_{n}(x)
$$

This implies that

$$
s_{n}(z)=-\frac{2}{2 z+s_{n}(z)}+\delta_{n}(z)
$$

In [8] it was proved that there exists some positive constant $C_{0}$ such that, for any $v \geqslant$ $C_{0} M^{1 / 2} n^{-1 / 2}, M=\sup _{l, j}\left\{\mathbf{E}\left|X_{l j}\right|^{4}, \mathbf{E}\left|Y_{l j}\right|^{4}\right\}$,

$$
\operatorname{Im}\left(z+\frac{\delta_{n}(z)}{2}\right)>0, \quad z=u+i v .
$$

For $z=u+i v$ such that $|u| \leqslant \sqrt{2}, v \geqslant C_{0} M^{1 / 2} n^{-1 / 2}$ we obtained the bound

$$
\left|\delta_{n}(z)\right| \leqslant \frac{C M^{1 / 2}}{n v} \text {. }
$$

(See $[8$, Lemmas 5.1, 5.2].) Solving the equation (4.8) and choosing a solution with $\operatorname{Im} s_{n}(z)>0$ we get, for $v \geqslant C_{0} M^{1 / 2} n^{-1 / 2}$

$$
s_{n}(z)=-\left(z-\frac{\delta_{n}(z)}{2}\right)+\sqrt{\left(z+\frac{\delta_{n}(z)}{2}\right)^{2}-2} .
$$

The equality (4.10) implies that, for $v \geqslant C_{0} M^{1 / 2} n^{-1 / 2}$

$$
\left|s_{n}(z)-s(z)\right| \leqslant\left|\delta_{n}(z)\right|\left(1+\left|\sqrt{\left(z+\frac{\delta_{n}(z)}{2}\right)^{2}-2}+\sqrt{z^{2}-2}\right|^{-1}\right)
$$

Since $\operatorname{Im} \sqrt{\left(z+\delta_{n}(z) / 2\right)^{2}-2}$ and $\operatorname{Im} \sqrt{z^{2}-2}$ are positive, we have, for $z=u+i v$ such that $u \in \mathbf{J}_{\varepsilon}^{\prime(r)} \cup \mathbf{J}_{\varepsilon}^{\prime(l)}$ and $v \geqslant C_{0} M^{1 / 2} n^{-1 / 2}$,

$$
\left|s_{n}(z)-s(z)\right| \leqslant\left|\delta_{n}(z)\right|\left(1+\left|\operatorname{Im}\left\{\sqrt{z^{2}-2}\right\}\right|^{-1}\right) .
$$

Note that

$$
\operatorname{Im} \sqrt{z^{2}-2}=\frac{\left|\operatorname{Im}\left(z^{2}-2\right)\right|}{\sqrt{2\left(\left|z^{2}-2\right|+\operatorname{Re}\left(z^{2}-2\right)\right)}}
$$


Furthermore, since $|u|^{2} \leqslant 2$ we have

$$
\operatorname{Re}\left(z^{2}-2\right) \leqslant 0
$$

and

$$
\left|z^{2}-2\right|^{2}=\left(u^{2}-2\right)^{2}+4 u^{2} v^{2}-2\left(u^{2}-2\right) v^{2}+v^{4} \leqslant 9\left(v^{2}+\eta^{2}\right) .
$$

In the last inequality we used that $v \leqslant 1$ and $0 \leqslant 2-u^{2} \leqslant 2 \eta$. The relations (4.12)-(4.14) together imply that

$$
\left|\operatorname{Im} \sqrt{z^{2}-2}\right|^{-1} \leqslant \frac{C\left(v^{2}+\eta^{2}\right)^{1 / 4}}{v} .
$$

The inequalities (4.15) and (4.11) together imply that

$$
\left|s_{n}(z)-s(z)\right| \leqslant C\left|\delta_{n}(z)\right| \frac{\left(\eta^{2}+v^{2}\right)^{1 / 4}}{v} \leqslant \frac{C\left(\eta^{2}+v^{2}\right)^{1 / 4}}{n v^{2}} .
$$

Using now the inequality (4.4) with $\eta=C n^{-1 / 3}, \varepsilon=C n^{-1 / 2}, v_{0}=C n^{-1 / 2}$ we get

$$
\Delta \leqslant \Delta_{\eta} \leqslant \frac{C \eta^{1 / 2}}{n v_{0}}+\frac{C}{n \sqrt{\eta}}+C v_{0} \sqrt{\eta} \leqslant C n^{-2 / 3} .
$$

If $\Delta \geqslant \Delta_{\eta}$, we use the nonuniform bound and obtain $\Delta \leqslant C n^{-2 / 3}$. These inequalities together imply that $\Delta \leqslant C n^{-2 / 3}$, which proves Theorem 1.2 .

\section{REFERENCES}

1. Bai Z. D. Convergence rate of expected spectral distributions of large random matrices. I: Wigner matrices. - Ann. Probab., 1993, v. 21, № 2, p. 625-648.

2. Bai $Z$. D. Methodologies in spectral analysis of large dimensional random matrices, a review. - Statist. Sinica, 1999, v. 9, № 3, p. 611-677.

3. Bai Z.D. Remarks on the convergence rate of the spectral distributions of Wigner matrices. - J. Theoret. Probab., 1999, v. 12, № 2, p. 301-311.

4. Erdélyi A. Asymptotic solutions of differencial equations with transition points or singularities. - J. Math. Phys., 1960, v. 1, p. 16-26.

5. Гирко В. Л. Случайные матрицы. Киев: Виша школа, 1975, 448 с.

6. Girko V.L. Convergence rate of the expected spectral functions of symmetric random matrices is equal to $O\left(n^{-1 / 2}\right)$. - Random Oper. Stochastic Equations, 1998, v. 6, № 4 , p. 359-408.

7. Гирко В.Л. Асимптотика распределения спектра случайных матриц. - Успехи матем. наук, 1989 , т. 44 , с. 7-34.

8. Götze F., Tikhomirov A. Rate of convergence to the semi-circular law. Preprint SFB343 00-125, Bielefeld: Bielefeld Universität, 2000; www.mathematik.unibielefeld.de/sfb343/

9. Mehta M. L. Random Matrices. Boston: Academic Press, 1991, 562 p.

10. Mehta M. L. Random Matrices and the Statistical Theory of Energy Levels. New York: Academic Press, 1967, 259 p.

11. Muckenhoupt B. Mean convergence of Hermite and Laguerre series. I, II. -- Trans. Amer. Math. Soc., 1970, v. 147, p. 419-431.

12. Пастур Л.А. Спектры случайных самосопряженных операторов. - Успехи матем. наук, 1973 , т. 28 , № 1, с. 3-64.

13. Wigner E. P. Characteristic vectors of bordered matrices with infinite dimensions. Ann. Math. (2), 1955, v. 62 , p. $548-564$.

14. Wigner E. P. On the distribution of the roots of certain symmetric matrices. - Ann. Math. (2), 1958, v. 67, p. $325-327$. 